\title{
BIOMETRIA DE FRUTOS E GERMINAÇÃO DE SEMENTES DE Couratari stellata A. C. Smith (LECYTHIDACEAE)
}

\author{
Eniel David CRUZ ${ }^{1}$ \& José Edmar Urano de CARVALHO ${ }^{1}$
}

\begin{abstract}
RESUMO - Estudou-se as características biométricas de frutos e quantificou-se a germinação em Couratari stellata, espécie cuja altura varia de 30 a $40 \mathrm{~m}$ e ocorre em solos de terra firme das Guianas até a região central e oeste da Amazônia. Determinou-se o comprimento e o número de sementes por fruto; número de sementes completamente formadas e de sementes vazias; o grau de umidade; a massa de 100 sementes; a percentagem de germinação, de plântulas anormais e de sementes mortas; e o tempo médio de germinação. A semeadura foi realizada em substrato de areia e serragem na proporção de $1: 1$, em quatro repetições de 50 sementes. Comprimento e número de sementes por fruto variaram de 59,0 a $97,0 \mathrm{~mm}$ e de cinco a 26 sementes. As percentagens de sementes completamente formadas e de sementes vazias foram de $93,3 \%$ e $6,7 \%$, respectivamente. A massa de 100 sementes foi de $10,7 \mathrm{~g}$ com $56,8 \%$ de umidade. A germinação foi lenta e desuniforme iniciando-se aos 29 dias após a semeadura, atingindo a germinação de $82,5 \%$ aos 65 dias. O tempo médio de germinação foi de 45,5 dias. A percentagem de sementes mortas e de plântulas anormais foi de $17,0 \%$ e $0,5 \%$, respectivamente. Não houve relação entre tamanho de frutos e número de sementes por fruto. Observou-se também que sementes de $C$. stellata apresentam dormência.
\end{abstract}

Palavras-chave: dormência, tempo médio de germinação, biometria de frutos

\section{Fruit biometry and seed germination of Couratari stellata A. C . Smith (Lecythidaceae)}

\begin{abstract}
This study evaluated the biometric characteristics of fruits and quantified seed germination of Couratari stellata, a 30-40 m tree species occurring on non-inundated soil from the Guianas to central western-Amazonia. The fruit length, number of seeds per fruit, number of completely formed and empty seeds, seed moisture, mass of 100 seeds, percentage of seed germination, mean time of germination, dead seeds and abnormal seedlings were evaluated. The seeds were sown in a substrate containing sand and sawdust $(1: 1)$, in four replications of 50 seeds. Fruit length ranged from 59 to $97 \mathrm{~mm}$ with five to 26 seeds. The percentage of completely formed seeds was $93.3 \%$ and empty seeds was $6.7 \%$. One hundred seeds weighed $10.7 \mathrm{~g}$, with $56.8 \%$ humidity. Germination was slow and not uniform, starting 29 days after sowing. Maximum germination, $82.5 \%$, was recorded 65 days after sowing. Mean time of germination was 45.5 days. Percentage of dead seeds and abnormal plants was $17.0 \%$ and $0.5 \%$, respectively. No relation was found between length of fruits and seed number per fruit. The seeds of C. stellata present dormancy.
\end{abstract}

Key-words: Dormancy, mean time of germination, fruit biometry

\section{INTRODUÇÃO}

O gênero Couratari é caracterizado por árvores com altura variando de 20 a $50 \mathrm{~m}$, sendo encontrado no Brasil, Colômbia, Costa Rica, Guianas, Panamá e Venezuela (Mori \& LepschCunha, 1995). No Brasil, as espécies desse gênero são encontradas nos Estados do Amapá, Amazonas, Mato Grosso e Pará (Camargos et al., 1996), sendo conhecidas como imbirema, tauari-amarelo, tauari-morrão e estopeiro (Souza et al., 1997).

No Estado do Pará, no período de 1987 a 1995 , foram exportados cerca de $255.000 \mathrm{~m}^{3}$

${ }_{1}^{1}$ Pesquisadores da Embrapa Amazônia Oriental, Caixa Postal 48, CEP 66017-970, Belém, PA, Brasil. E-mail: eniel@cpatu.embrapa.br 
de madeiras de espécies do gênero Couratari (Carvalho, 1996). Souza et al. (1997) citam que a cor da madeira das espécies desse gênero varia de branco-amarelado a marron-amareladoclaro, com densidade de 590 a $1.100 \mathrm{~kg} / \mathrm{m} 3$, podendo ser utilizada na construção civil e naval, móveis, artigos decorativos, utensílios domésticos, instrumentos musicais, embalagens, marcenaria, compensados e outros.

Couratari stellata A. C. Smith é uma espécie, cuja altura varia de 30 a $40 \mathrm{~m}$, ocorrendo em solos de terra firme, das Guianas até a região central e oeste da Amazônia (Mori \& Lepsch-Cunha, 1995). A floração ocorre de setembro a dezembro, produzindo centenas de flores durante um a dois meses (Mori \& Prance, 1987), sendo os frutos deiscentes, cujas sementes são aladas e dispersas pelo vento (Mitchell \& Mori, 1987).

A caracterização biométrica de frutos pode fornecer importante informação de modo a permitir diferenciar espécies do mesmo gênero no campo, como ocorre com a Hymenaea courbaril que tem frutos cerca de quatro vezes maior que os frutos de $\mathrm{H}$. intermedia (Cruz et al., 2001).

Segundo Baskin \& Baskin (1998), informações sobre a germinação das sementes são importante para entender as estratégias reprodutivas das espécies. $\mathrm{O}$ conhecimento sobre o processo germinativo das sementes de espécies florestais é importante para subsidiar trabalhos de regeneração, silvicultura e conservação e utilização de recursos genéticos.

O objetivo deste trabalho foi caracterizar frutos e quantificar a germinação de sementes de C. stellata.

\section{MATERIAL E MÉTODOS}

\section{Local de colheita e de avaliação}

Os frutos foram coletados de uma árvore, em floresta nativa, no município de Paragominas, Pará (0257’56"S e 4740’21"O). A colheita deu-se na ocasião da abertura do opérculo e da disseminação das sementes. As avaliações foram realizadas na Embrapa Amazônia Oriental, Belém, Pará, Brasil, dois dias após os frutos terem sido coletados.

\section{Biometria de frutos}

Foram determinados o comprimento, medido no sentido longitudinal do fruto (Fig. 1) e o número de sementes por fruto, em 100 frutos; as percentagens de sementes completamente formadas e vazias, em 980 sementes; a massa de 100 sementes, em oito repetições com a mesma quantidade de sementes; e o grau de umidade das sementes, em quatro repetições de dez sementes, através de estufa a $105 \pm 3^{\circ} \mathrm{C}$, durante 24 horas, de acordo com Brasil (1992).

\section{Germinação de sementes}

O teste de germinação foi realizado nas condições ambientais de Belém (temperatura média de $27,5^{\circ} \mathrm{C}$ e $84 \%$ a média da umidade relativa do ar), após uma pré-secagem das sementes por 24 horas em ambiente com $55 \pm 5 \%$ de umidade relativa do ar e $25 \pm 2^{\circ} \mathrm{C}$ de temperatura, visando uniformizar a umidade das mesmas. As sementes foram semeadas em substrato constituído de areia e serragem na proporção volumétrica de $1: 1$, previamente esterilizado em água quente por duas horas, em quatro repetições de 50 sementes. Durante o período de avaliação, o substrato foi irrigado a cada dois dias. O número de sementes germinadas foi quantificado diariamente e, no final do experimento, foram computados o número de sementes mortas e de plântulas anormais. O tempo médio de germinação foi estimado segundo Edmond \& Drapala (1965), pela equação:

$$
\mathrm{Tm}=\frac{\mathrm{G}_{1} \mathrm{~T}_{1}+\mathrm{G}_{2} \mathrm{~T}_{2}+\ldots+\mathrm{G}_{n} \mathrm{~T}_{n},}{\mathrm{G}_{1}+\mathrm{G}_{2}+\ldots+\mathrm{G}_{n}}
$$

onde, Tm é o tempo médio necessário para atingir a germinação máxima; $\mathrm{G}_{1}, \mathrm{G}_{2}$ e $\mathrm{G}_{\mathrm{n}}$ é o número de sementes germinadas nos tempos $\mathrm{T}_{1}, \mathrm{~T}_{2}$ e $\mathrm{T}_{\mathrm{n}}$, respectivamente. Considerou-se como germinadas as sementes que deram origem a plântulas normais, ou seja, com todas as suas estruturas essenciais bem desenvolvidas, completas, proporcionais e sadias. Determinouse, também, as percentagens de sementes mortas e de plântulas anormais, as quais foram identificadas conforme Brasil (1992). 

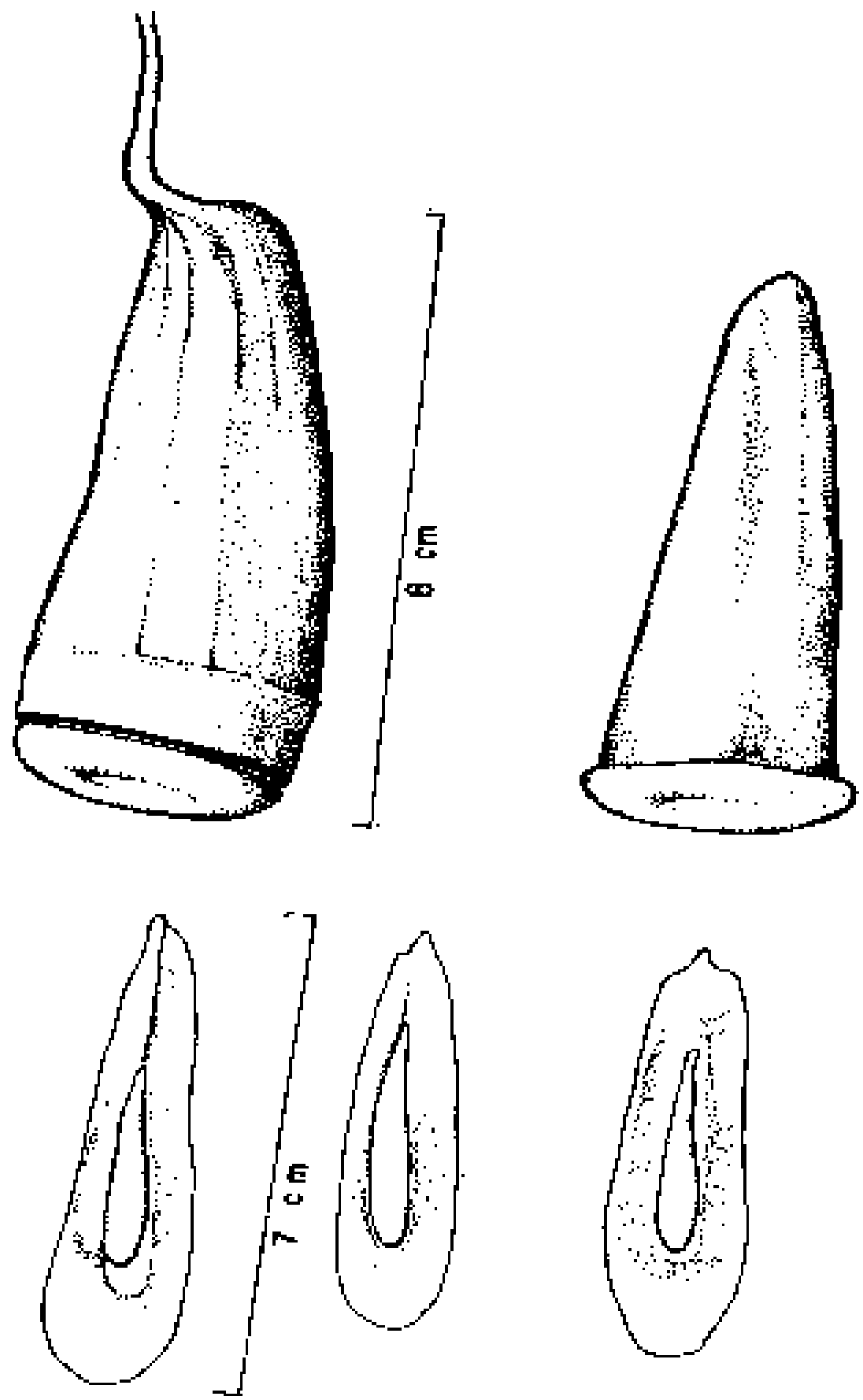

Figura 1 - Fruto tipo pixídio (A), eixo do fruto (B) e sementes aladas (C) de Couratari stellata. Ilustração: Elielson Rocha. 


\section{Análise estatística}

Analisou-se os dados através de distribuição de freqüência e de análise de regressão. A análise de regressão foi efetuada através do software Statistica for Windows (Statsoft, 1998).

\section{RESULTADOS E DISCUSSÃO}

\section{Biometria de frutos}

O fruto de tauari (C. stellata) (Fig.1A) é um pixídio, cujo comprimento variou de 59 a

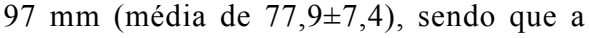

maioria apresentou comprimento variando de 71 a $80 \mathrm{~mm}$ (Fig. 2A), menores que os frutos de Couratari cf. macrosperma e de $C$. martiana, conforme descrição destas espécies apresentadas por Deus et al. (1993) e Corrêa (1978), respectivamente. O número de sementes por fruto oscilou de cinco a 26 sementes (média de 9,8 83,0 ), predominando frutos com seis a nove sementes (Fig. 2B). A percentagem de sementes completamente formadas foi $93,3 \%$ e de sementes vazias $6,7 \%$. Não foi observada relação entre número de sementes por fruto e tamanho dos frutos $\left(Y=2,807+0,09 X ; R^{2}=0,038\right)$.
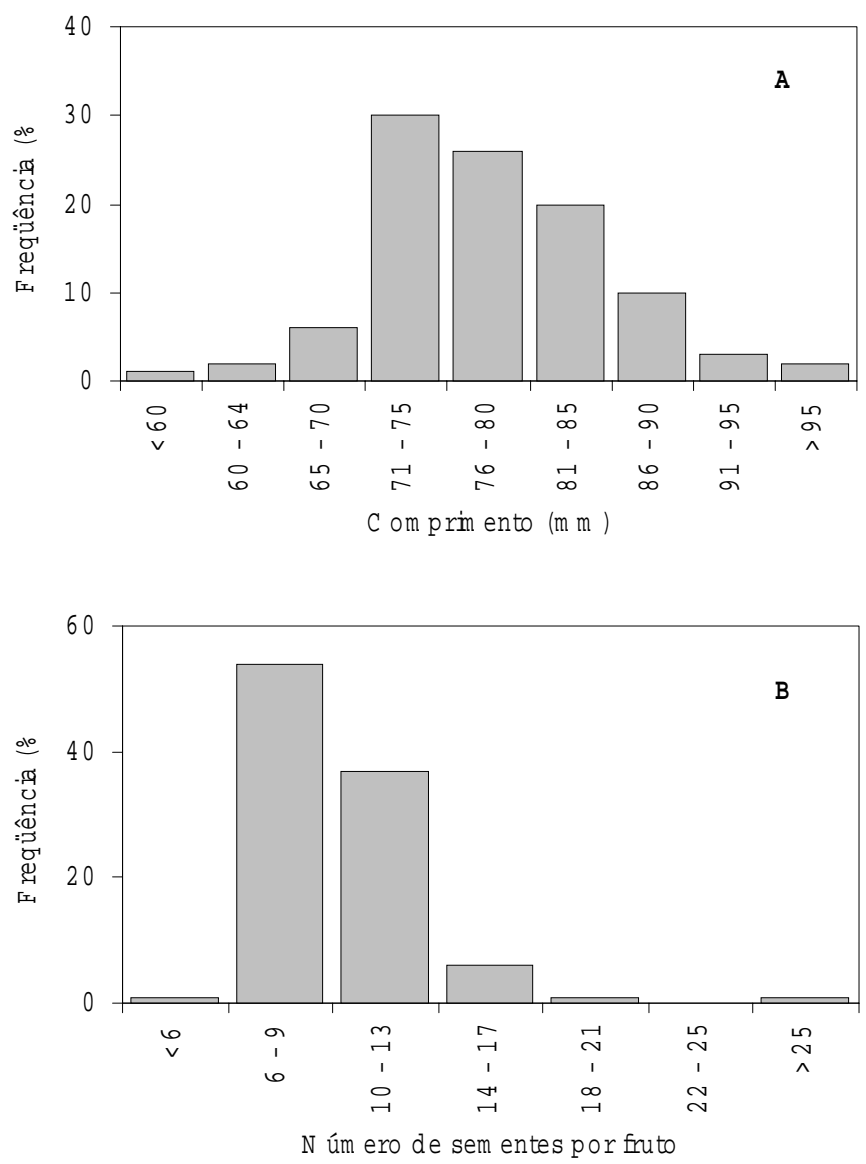

Figura 2 - Histograma referente ao comprimento de frutos (A) e número de sementes por frutos (B), em Couratari stellata. 


\section{Germinação de sementes}

O grau de umidade das sementes, imediatamente após a retirada dos frutos, foi de $56,8 \%$, reduzindo para $17,1 \%$ após a permanência de 24 horas em ambiente com temperatura média de $25 \pm 2^{\circ} \mathrm{C}$ e $55 \pm 5 \%$ de umidade relativa do ar média. Em sementes com $56,8 \%$ de umidade a massa de 100 sementes foi de $10,7 \mathrm{~g}$.

A germinação de sementes de $C$. stellata é do tipo epigeal e as plântulas fanerocotiledonares. O processo de germinação foi relativamente lento e desuniforme, iniciando 29 dias após a semeadura (Fig. 3), fatos estes também observados por Reis et al. (1979) em Bertholletia excelsa (Lecythidaceae). O final da germinação ocorreu aos 65 dias após a semeadura, quando foi atingido o percentual de $82,5 \%$, valor superior ao registrado por Alencar \& Magalhães (1979) em Cariniana micrantha (Lecythidaceae). A curva de germinação é do tipo sigmoidal e segue o modelo padrão de germinação da maioria das espécies descritas por Tipton (1984), apresentando um período inicial, onde houve um pequeno aumento na percentagem de germinação, seguido por um período de grande aumento na germinação e por outro, caracterizado por aumentos decrescentes nas taxas de germinação. Para Ng (1978), sementes de espécies arbóreas, que requerem mais que 12 semanas para germinarem na sua totalidade, apresentam algum tipo de dormência e, Harsh \& Ojha (2000) citam que muitas espécies arbóreas possuem graus de dormência ou têm problemas na germinação. A ocorrência de dormência nas sementes, provocando germinação lenta e irregular, pode causar restrição a um manejo eficiente na sementeira. Entretanto, para Mohamed-Yasseen et al. (1994), a dormência também é reconhecida por proteger a semente das flutuações de temperatura, umidade e da incidência de microorganismos. O tempo médio de germinação foi de 45,5 dias, superior ao obtido em Lecythis pisonis, da mesma família, por Carvalho et al. (1998). No final do teste de germinação, observou-se que $17,0 \%$ das sementes estavam

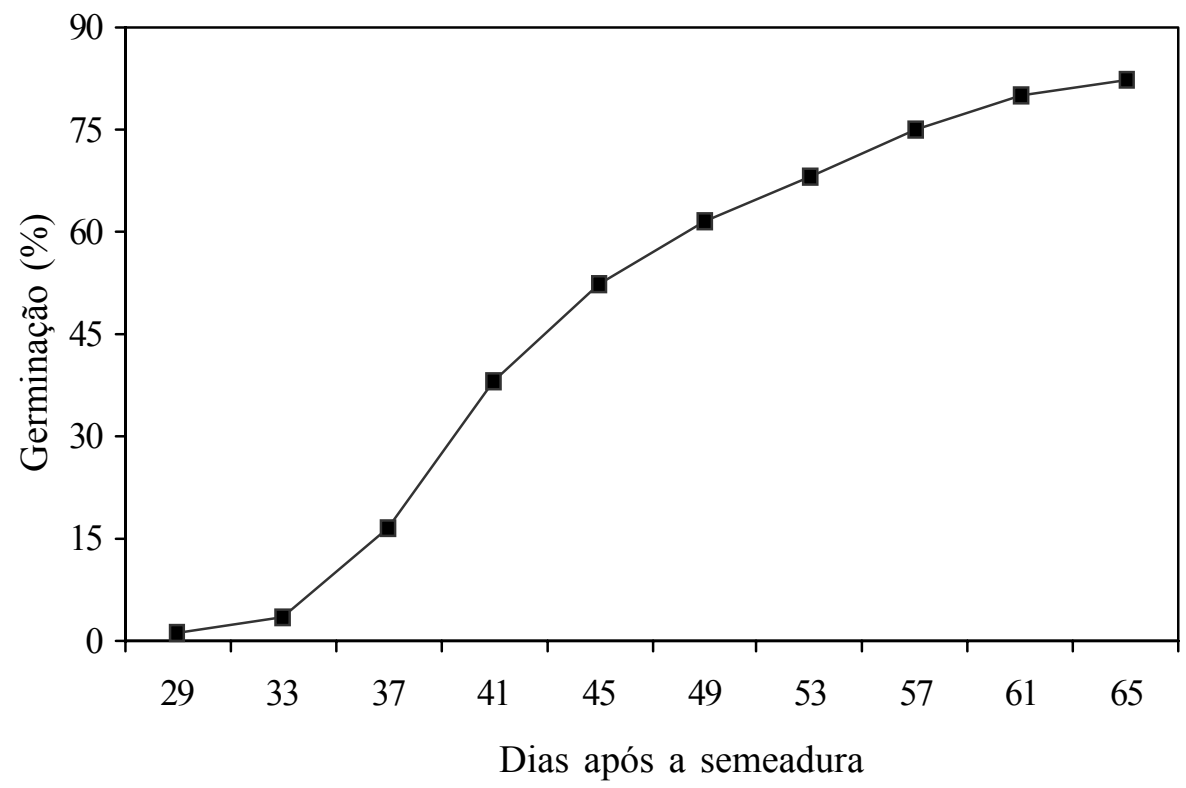

Figura 3 - Germinação de sementes de Couratari stellata com 17,1\% de umidade. 
mortas e que $0,5 \%$ produziram plântulas anormais.

\section{CONCLUSÃO}

Concluiu-se que, na amostra colhida de C. stellata, o número de sementes por fruto foi independente do tamanho do fruto e, também, que as sementes apresentaram germinação desuniforme, indicando a existência de dormência.

\section{BIBLIOGRAFIA CITADA}

Alencar, J. da C.; Magalhães, L.M.S. 1979. Poder germinativo de sementes de doze espécies florestais da região de Manaus. Acta Amazonica, 9(3): 411-418.

Baskin, C.C.; Baskin, J.M. 1998. Seeds: ecology, biogeography, and evolution of dormancy and germination. Academic Press, London, 666p.

Brasil. 1992. Regras para análise de sementes. Ministério da Agricultura e Reforma Agrária. Brasília, 365p.

Camargos, J.A.A.; Czarneski, C.M.; Meguerditchian, I.; Oliveira, D. de. 1996. Catálogo de árvores do Brasil. Instituto Brasileiro do Meio Ambiente e dos Recursos Naturais Renováveis, Brasília, $887 \mathrm{p}$.

Carvalho, J.E.U.; Nascimento, W.M.O.; Müller, C.H. 1998. Características físicas e de germinação de sementes de espécies frutiferas nativas da Amazônia. Boletim de Pesquisa 203, Embrapa Amazônia Oriental, Belém, 18p.

Carvalho, G. dos S. 1996. Mapa comparativo das exportações de madeira do Estado do Pará e Amapá. Associação das Indústrias Exportadoras de Madeira do Estado do Pará, Belém, 22p.

Corrêa, M.P. 1978. Dicionário das plantas úteis e das exóticas cultivadas. Vol. VI. Imprensa Nacional, Rio de Janeiro, 777p.

Cruz, E.D.; Martins, F. de O.; Carvalho, J.E.U. de. 2001. Biometria de frutos e germinação de sementes de jatobá-curuba (Hymenaea intermedia Ducke, Leguminosae - Mimosoideae). Revista Brasileira de Botânica, 24(2): 161-165.

Deus, C.E. de.; Weigand Júnior, R.; Kageyama, P.Y.; Viana, V.M.; Ferraz, P. de A.; Borges, H.B.N.; Almeida, M.C.; Silveira, M.; Vicente, C.A.R., Andrade, P.H.C. 1993. Comportamento de 28 espécies arbóreas tropicais sob diferentes regimes de luz em Rio Branco, Acre. Universidade Federal do Acre, Rio Branco, 173p.

Edmond, J.B.; Drapala, W.J. 1965. The effects of temperature, sand and soil, and acetone on germination of okra see d. Proceedings of the American Jornal Society for Horticultural Science, 71: 428-434.

Harsh, N.S.K.; Ojha, B.M. 2000. A possible pretreatment for seeds of tropical species. Seed Science and Technology, 28(2): 512516.

Mohamed-Yasseen, Y.; Barringer, S.A.; Splittstoesser, W.E.; Costanza, S. 1994. The role of seed coats in seed viability. Botanical Review, 60(4): 426-439.

Mori, S.A.; Lepsch-Cunha, N. 1995. The Lecythidaceae of a Central Amazonian moist forest. Memoirs of the New York Botanical Garden, 75: 1-55.

Mori, S.A.; Prance, G.T. 1987. Phytogeography. Memoirs of the New York Botanical Garden, 44: 55-71.

Mitchell, J.D.; Mori, S.A. 1987. Ecology. Memoirs of the New York Botanical Garden, 44: 113-123.

Ng, F.S.P. 1978. Tropical trees as living systems. In: Tomlinson, P.B.; Zimmermann, M.H. (Eds.). Strategies of establishment in Malayan forest trees. Cambridge University Press, Cambridge, p.129-162.

Reis, G. dos S.; Carvalho, J.E.U. de; Müller, C.H.; Figueiredo, F.J.C. 1979. Calibração do teste de tetrazólio em sementes de castanha-do-brasil. Comunicado Técnico 17, Embrapa-CPATU, Belém, 9p.

Souza, M.H. de; Magliano, M.M.; Camargos, J.A.A.; Souza, M.R. de. 1997. Madeiras 
tropicais brasileiras. Instituto Brasileiro do Meio Ambiente e dos Recursos Naturais Renováveis, Brasília, 151p.

Statsoft. 1998. Statistica for Windows. General conventions and statistics. Statsoft, Tulsa, OK inc.

Tipton, J.L. 1984. Evaluation of three growth curve models for germination data analysis. Journal of the American Society for Horticultural Science, 109:451-454.

Recebido: $16 / 10 / 2001$

Aceito: 23/09/2002 
